\title{
Integrating Face and the Both Irises for Personal Authentication
}

\author{
Leila Zoubida, Réda Adjoudj \\ EEDIS Laboratory, Djillali Liabes University of Sidi Bel Abbes, Sidi Bel Abbes, 22000, Algeria \\ E-mail: leilaz31@yahoo.fr, AdjReda@yahoo.fr
}

\begin{abstract}
The biometric authentication, which use the characteristic of persons to verify their identity by using their behavioral and physiological characteristics are an important application of the pattern recognition. There are different biometric modalities used to achieve the task of recognition. Among the most popular traits biometric currently used in several applications are the face and the iris. This paper proposes a multi-biometric technique which combines the face modality with the both irises (the left and the right irises) to authenticate the persons. The fusion of these two traits biometrics combines the advantages of the both instances of the iris modality with the face modality. The wavelets are used for the extraction of the biometrics features and the Support Vector Machine is used to obtain scores for fusion. Then, the Min-Max operator is used to normalize these scores. The fusion is operated at score level by the combination of two methods: a combination method and a classification method. So, we used the five rules (Sum, Product, Max, Min, Mean) combined with a classification method for the fusion. The Fusion is tested using the SDUMLA-HMT database. The experimental results show that multi-biometric systems achieve the task of recognition better than the mono-modal systems.
\end{abstract}

Index Terms-Multibiometric, Pattern Recognition, Iris Authentication, Face Authentication, Score Level Fusion, Support Vector Machines.

\section{INTRODUCTION}

Today, biometric is considered as one of the important applications of the pattern recognition, which solves many problems of security. It refers to the automatic recognition of persons based on their traits biometrics.

The biometric systems based on one biometric modality, suffer from a number of limitations like the [1]:

- Non-universality,

- Possibility of fraud,

- Limitation in terms of performance recognition.

In contrast, the integration of the biometric information presented by the various modalities can solve many problems of the mono-modal systems. However, there are several biometric modalities such as:
- Iris,

- Face,

- Fingerprints,

- Hand,

- Voice,

- Retinal pattern,

- $\quad \ldots$.

The biometric system can operate in one of these two modes:

- The Authentication mode,

- The Identification mode.

In this work, we integrate two modalities which are: the face and the both instances of the iris modality. Our multi-biometric system operates in the authentication mode. We choose the fusion of these two modalities because the face modality is no-intrusive [2] and it considered as one of the most natural ways to recognize a person. In addition, the iris is one of the most accurate biometric modalities [3] and its falsification is difficult [4]. Also, the acquisition of the both modalities can be done by the same capture device.

This paper is based on our previous work which fused the both units of the iris modality [5]. The present paper proposes a multi-biometric technique based on the both irises and the face fused at score level.

The rest of the paper is organized as follows. The face authentication and the iris authentication are presented in Section II. Section III describes the steps of our approach. The experimental results obtained are presented in Section IV and an overall conclusion is presented in Section V.

\section{FACE AUthentiCATION AND IRIS AUTHENTICATION}

The multimodal system based on the fusion of the iris and the face is a biometric research that is considered to be an important part of the biometrics techniques. The integration of the biometric information can operate before or after the matching step.

The fusion of the face and the iris modality has attracted the attention of several researchers:

- Before the matching step: 
- The feature level: Rattani and Tistarelli [6]; Son and lee [7]; Lin and al. [8]; Gan and al. [9]; Gan and liu [10]; Fakhar and al. [11]; Chen and Te Chu [12]; Wang and al. [13]; Kekre and al. [14]; Sharma and Kumar [15]; Nair and al. [16]; Wang and al. [17]; Abuguba and al. [18].

- After the matching step:

- The score level: Zhang and al. [19]; Wang and al. [20]; Morizet and Gilles [21]; Zhang and al. [22]; Kim and al. [23]; Eskandari and Toygar [24] [25]; Eskandari and al. [26]; Liau and Isa [27]; Vasta and al. [28]; Wang and Han [29]; Johnson and al. [30]; Wang and al. [31].

- The rank level: Connaughton and al. [32] [33].

- The decision level: Lee and al. [34].

The acquisition and the processing of the biometric data are two important requirements in the multimodal systems. These two requirements can operate successively (the serial mode) or simultaneously (the parallel mode).

The parallel mode is the most used because it offers the possibility of use of all available biometric information, so that allow improving the performance of the fusion.

The parallel mode is used in this work, as shown in Fig.1:

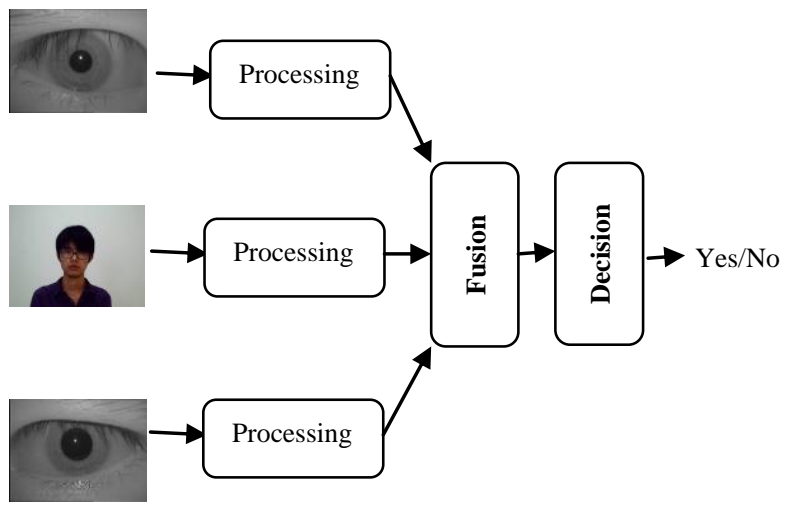

Fig.1. The architecture of the multi-biometric system with the parallel mode.

\section{PROPOSED APPROACH}

In this section, we will describe the steps of our approach used for the multi-biometric technique. It is based on the fusion of the biometric characteristics which are: the face; the right and the left irises.

For the both modalities (the face and the iris), the wavelets are used for the feature extraction. The matching score for each modality is calculated by using the SVM method and the scores are normalized by the Min-Max method. Then, the final score is generated by using the combination of two methods which are: the combination method and the classification method [35], which is then passed to the decision module.

Our multi-biometrics system is developed using two traits (the face and the iris (the left iris and the right iris)). The structure of our multimodal biometric technique is shown in Fig. 2.

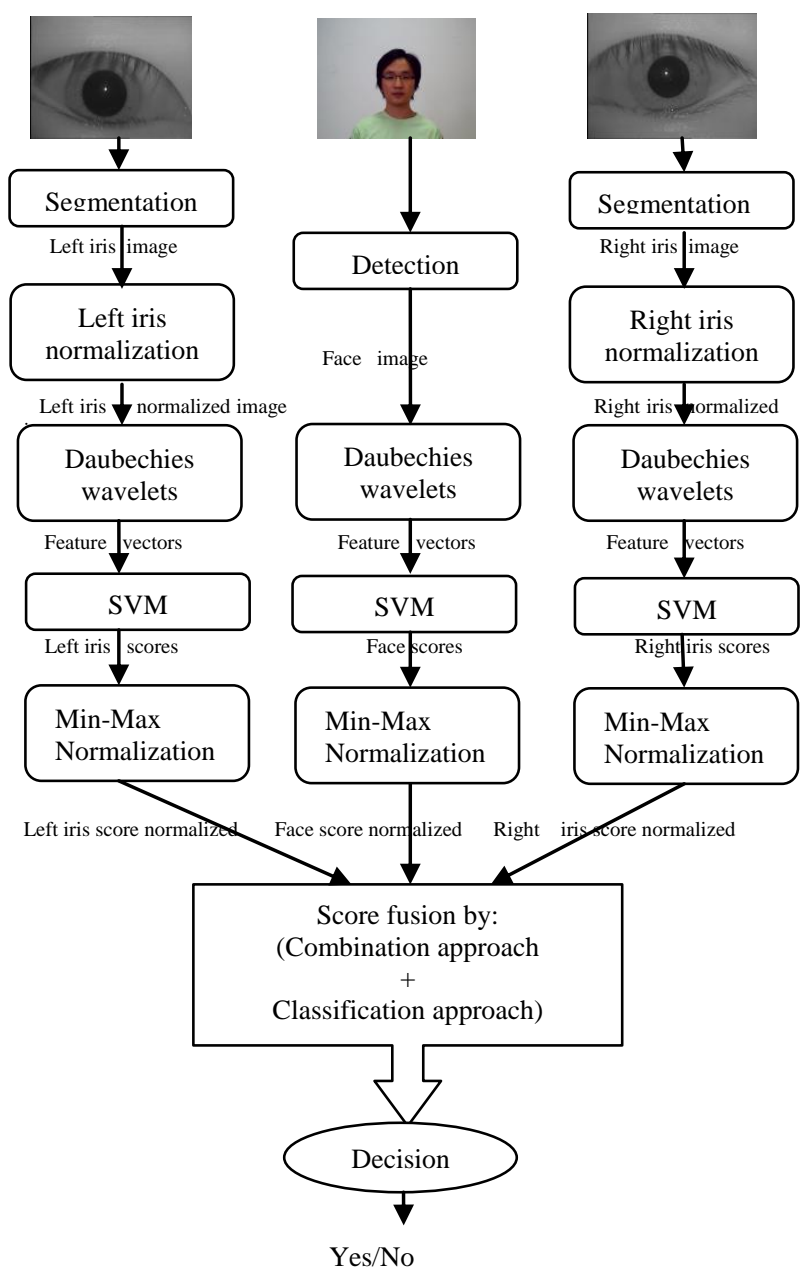

Fig.2. System structure

The steps used in our approach are:

1. Capture: We used the SDUMLA-HMT database [36] in order to test the performance of our system. This database is the most used database in this field of research, see details in section IV.

2. Detection: It is necessary to segment the image to isolate the face and the iris from the rest of the image. The face is detected manually and the detection of the iris is passed by two steps: the segmentation and the normalization.

a. Segmentation: in this step, we used a modified method of the method used in the Masek system [37], in order to isolate the iris from the rest of the image. The system Masek [37] segments the iris by using the Hough transform method which localized the pupil, the iris, the eyelids and the eyelashes. Also, The Hough Transform determined 
the radius and the coordinates of the center of the both circles (the pupil and the iris). In our work, the same steps as the Masek system [37] are followed for the process of the iris segmentation, but we proposed that the coordinates of the center of the pupil and the iris are the same [5], it isn't like the system Masek [37]. This last system segment the iris in the following manner [37]: The canny detection algorithm is used to generate the contours. Therefore, the white-iris border is detected by the vertical gradients, the pupil border is detected by the both gradients (vertical and horizontal), and the detection of the iris is done before that the pupil. In our work, we add a step after this last step [5].

We found that there are several examples of incorrect iris segmentation by the application of the Masek system on the SDUMLA-HMT iris database. However, the points of the pupil border are well detected [5]. This is what led us to propose that the two circles of the pupil and the iris are concentric. By using the Masek system, we can obtain the radius of the iris and the coordinates of the pupil center. Therefore, we used these parameters for the generation of a new circle of the iris. So, this new circle has the same center as the pupil detected previously and his radius is the same radius as the iris detected previously [5].

Figure 3 is a diagram that illustrates the steps of our modified method which is used for the process of the iris segmentation.

Figure 4 illustrates the difference between the same example of iris segmented by the Masek method [37] and the modified method [5]. However, while we were applying this modified method on the SDUMLA-HMT iris database, we found that the number of the poorly detected iris is reduced [5] compared to the Masek system. The other steps for the detection of the eyelids, the eyelashes, and the reflections are the same as the Masek system [37].

b. Normalization: In this step, we used the same method as the Masek system [37] to transform the irregular disc of the iris in a rectangular image of constant size. This system based on the normalization method "Pseudo-Polar" [38].

3. Feature extraction: In this step, we chose to use the same method for the feature extraction of the both modalities (the face and the both irises). Therefore, the single level two-dimensional wavelet decomposition of the signal using the Daubechies wavelet is used to extract the feature vectors of the face and the both units of irises.

At this level, we have three features vectors: the face features vector, the left iris features vector and the right iris features vector.

4. Obtain scores: The method of support vector machines (SVM) is used to get scores which are then used for the fusion. Our system is an authentication system which has as results authenticate or non-authenticate identity. Therefore, the SVM used is the binary SVM. There are several kernel functions which can use in the SVM. Among these kernel functions, we chose to use the linear function.

At this level, we have three scores: the score of the face, the score of the left iris and the score of the right iris.

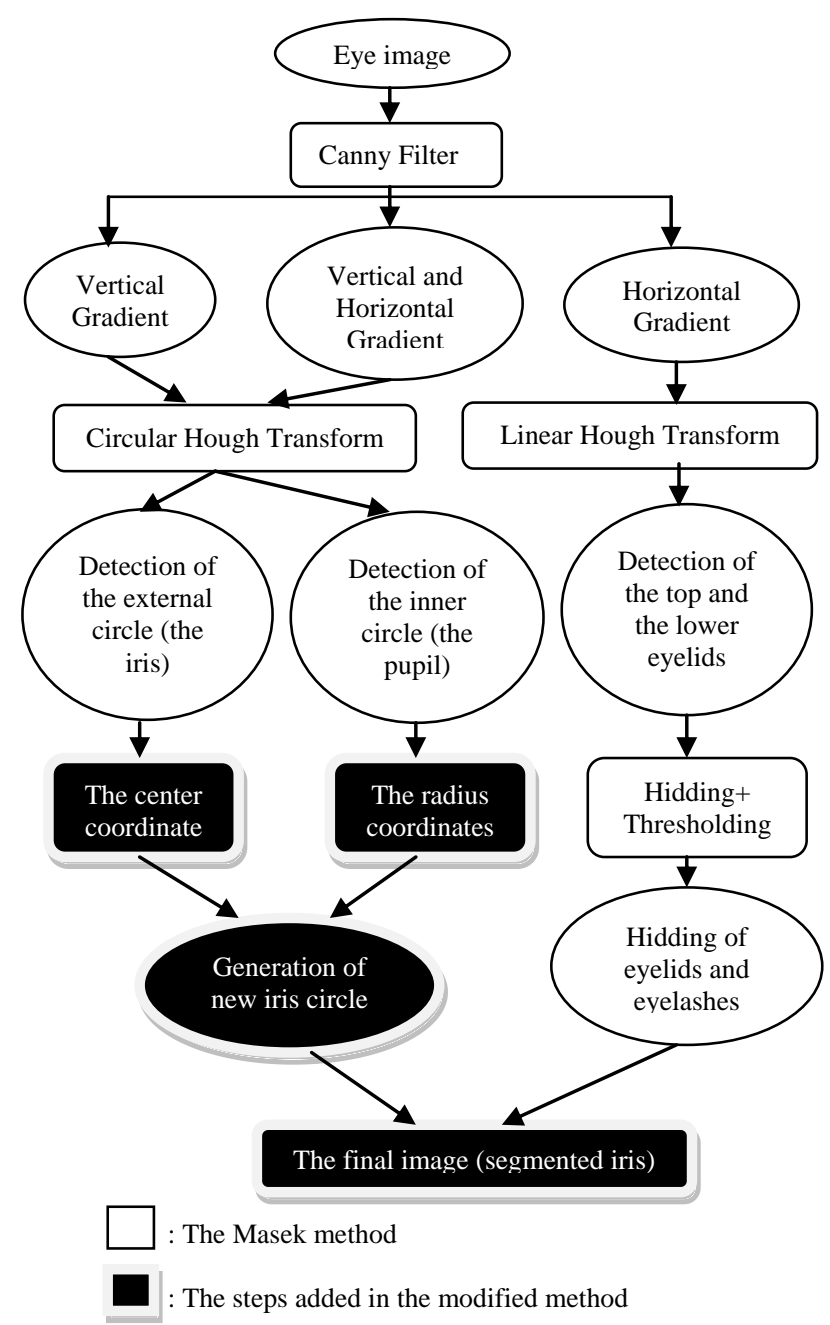

Fig.3. The diagram of the iris segmentation.

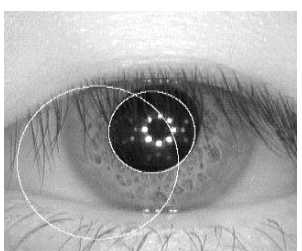

(a)

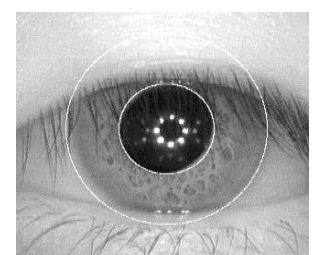

(b)
Fig.4. The same Example of iris segmented by: (a): The Masek method; (b): The modified method.

5. Normalized scores: There are different normalization techniques like Min-Max, Tanh and Z-score [39] [40]. In this work, the Min-Max 
normalization method is used to transform the mono-modal scores in a comparable range.

At this level, we have three normalized scores: the face normalized score, the left iris normalized score and the right iris normalized score.

6. Score fusion by the combination of two methods: the combination method and the classification method [35].

a. Combine scores: In this work, we use the five rules (Sum, Product, Min, Max and Mean) to form a single score which isn't compared with a threshold to take the final decision but it considered as a classification problem.

b. Classify the combined scores: The combined scores obtained in the previous step are used as inputs of the classification method [35]. It is a separable classification method which constructed differently the optimal hyper-plane and the maximum margin compared to the support vector machines [35].

The algorithm of this method [35] is also used in our previous work [5]. The steps of this algorithm are detailed in [5] as follow:

- The equation of the separating hyper-plane with maximum margin is written as:

$$
a y+b=0
$$

- Let: $\mathrm{Y}_{+}=\{\mathrm{y}: \mathrm{ay}+\mathrm{b} \geq 1\}$ and $\mathrm{Y}_{-}=\{\mathrm{y}: \mathrm{ay}+\mathrm{b} \leq-1\}$.

- Note that: $y_{+}$an element of $Y_{+}$and $y_{-}$an element of $\mathrm{Y}$.

$$
\begin{aligned}
& \underset{\mathrm{y}_{+} \in \mathrm{Y}_{+}}{\operatorname{Inf}}\left\|\mathrm{y}_{+}-\mathrm{y}_{-}\right\|=\left\|\overline{\mathrm{y}}_{+}-\overline{\mathrm{y}}_{-}\right\| \\
& \mathrm{y}_{-} \in \mathrm{Y}_{-}
\end{aligned}
$$

\section{$\left(\overline{\mathrm{y}}_{+}, \overline{\mathrm{y}}_{-}:\right.$Supports vectors $)$}

- $\bar{y}_{+}$and $\bar{y}_{-}$are used to find the parameters (a and b) of the separating hyper-plane:

$$
\begin{aligned}
& \mathrm{a}=\left(\bar{y}_{+}-\overline{\mathrm{y}}_{-}\right) \\
& \left\{\begin{array}{l}
\overline{a y}_{+}+b=1 \\
\overline{a y}_{-}+b=-1
\end{array} \Rightarrow b=-\frac{1}{2} a\left(\bar{y}_{+}+\bar{y}_{-}\right)\right. \\
& \Rightarrow b=-\frac{1}{2}\left(\bar{y}_{+}-\bar{y}_{-}\right)\left(\bar{y}_{+}+\bar{y}_{-}\right)=\frac{\left\|\bar{y}_{-}\right\|^{2}-\left\|\bar{y}_{+}\right\|^{2}}{2}
\end{aligned}
$$

$$
\begin{aligned}
& \tilde{\mathrm{Y}}_{+}=\left\{\mathrm{y}_{+} \in \mathrm{Y}_{+}:\left(\overline{\mathrm{y}}_{+}-\overline{\mathrm{y}}_{-}\right) \mathrm{y}_{+}+\frac{\left\|\overline{\mathrm{y}}_{-}\right\|^{2}-\left\|\overline{\mathrm{y}}_{+}\right\|^{2}}{2} \leq 1\right\} \\
& \tilde{\mathrm{Y}}_{-}=\left\{\mathrm{y}_{-} \in \mathrm{Y}_{-}:\left(\overline{\mathrm{y}}_{+}-\overline{\mathrm{y}}_{-}\right) \mathrm{y}_{-}+\frac{\left\|\overline{\mathrm{y}}_{-}\right\|^{2}-\left\|\overline{\mathrm{y}}_{+}\right\|^{2}}{2} \geq-1\right\}
\end{aligned}
$$

- $\quad$ Note that: $\quad \overline{\mathrm{y}}_{+} \in \tilde{\mathrm{Y}}_{+}, \quad \overline{\mathrm{y}}_{-} \in \tilde{\mathrm{Y}}_{-}$

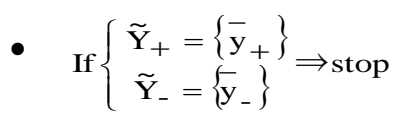

- Else,

$$
\begin{aligned}
& \text { If card }\left(\tilde{\mathrm{Y}}_{+}\right) \geq \operatorname{card}\left(\tilde{\mathrm{Y}}_{-}\right) \Rightarrow\left\{\begin{array}{l}
\operatorname{Max}\left\{-\|\mathrm{a}\|^{2}\right\} \\
\mathrm{a}\left(-\mathrm{y}_{+}+\overline{\mathrm{y}}_{+}\right) \leq 0 \\
\mathrm{a}\left(\mathrm{y}_{-}-\overline{\mathrm{y}}_{+}\right) \leq 1 \\
\mathrm{y}_{+} \in \tilde{\mathrm{Y}}_{+}, \mathrm{y}_{-} \in \tilde{\mathrm{Y}}_{-}
\end{array}\right. \\
& \text {If } \operatorname{card}\left(\tilde{\mathrm{Y}}_{+}\right)<\operatorname{card}\left(\tilde{\mathrm{Y}}_{-}\right) \Rightarrow\left\{\begin{array}{l}
\operatorname{Max}\left\{-\|\mathrm{a}\|^{2}\right\} \\
\mathrm{a}\left(\mathrm{y}_{-}-\mathrm{y}_{-}\right) \leq 0 \\
\mathrm{a}\left(\overline{\mathrm{y}}_{-}-\mathrm{y}_{+}\right) \leq-1 \\
\end{array}\right.
\end{aligned}
$$

The maximization of a concave quadratic program is the result of the SVM. This quadratic program can be solved by the projection method [41]. The projection of a point $0 \in R^{n}$ on the hyper-plane $\mathrm{a}\left(\mathrm{y}_{-}-\overline{\mathrm{y}}_{+}\right)=1$ is given by:

$$
P_{a}\left(y_{-}-\bar{y}_{+}\right)=-1(0)=0-\frac{1}{\left\|\left(y_{-}-\bar{y}_{+}\right)\right\|^{2}}\left(y_{-}-\bar{y}_{+}\right)
$$

For more details of this method see [41].

7. Decision: The decision can be taken according to the result of the classification method. The decision function is given by:

$$
\mathrm{G}(\mathrm{z})=\operatorname{sign}(\mathrm{az}+\mathrm{b})
$$

(sign: is the sign of the result of $(a z+b), \mathrm{z}$ : is an unknown element to classify, and ( $a$ and $b$ ) are the parameters which are determined by the classification method [35]). If the output of the function is positive then the person is authenticated. Otherwise, if the output is negative then the claimed identity is rejected [5].

- Let: 


\section{EXPERIMENTAL RESULTS}

\section{A. Description of the Database}

The integrated biometric system was tested on the nonchimerical database (SDUMLA-HMT). This database contains the both irises (the left and the right irises) with the face modality.

The SDUMLA-HMT was collected by Shandong University, Jinan, China in 2010. This database is characterized by: [42]

- The number of subjects in this database is 106 subjects, containing 45 females and 61 males which their age is between 17 and 31 .

- It includes 5 sub-databases:
- Face database;
- Iris database;
- Fingerprint database;
- Finger vein database;
- Gait database.

In the five sub-databases, all the biometric modalities with the same person are captured from the same subject [42].

In our work, we use the two sub-databases: The face database and the iris database, which will be detailed herein after:

- Face database: In this sub-database, seven ordinary digital cameras were used to capture the face. There are four variations in this database: [42]

○ 3 types of poses:

- Look upward,

- Forward,

- Downward

- 4 types of expressions:

- Smile,

- Frown,

- Surprise,

- Close eyes.

- 2 types of accessories:

- Glasses,

- Hat.

○ 3 types of illuminations:

- Illumination one,

- Illumination two,

- Illumination three.

So, the face database is composed of $7 \times(3+4+2+3) \times 106=8,904$ images.
The size of each image of this database is $640 \times 480$ pixels and its format is the "BMP" format. The total size of this database is $8.8 \mathrm{G}$ Bytes [42].

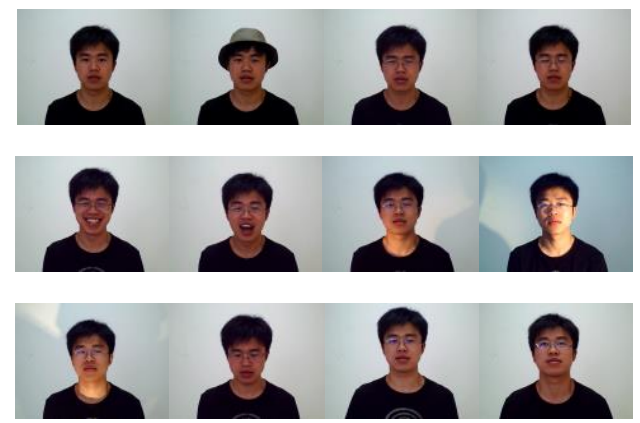

Fig.5. Examples of the third samples of the first subject for each variation.

- Iris database: The iris database contains 106 subjects and every subject provided 5 images for each of the eyes. So, the iris database is composed of $2 \times 5 \times 106=1,060$ images. The size of each image of this database is $768 \times 576$ pixels and it is saved in "BMP" format. The total size of the iris database is about $0.5 \mathrm{G}$ Bytes [42].
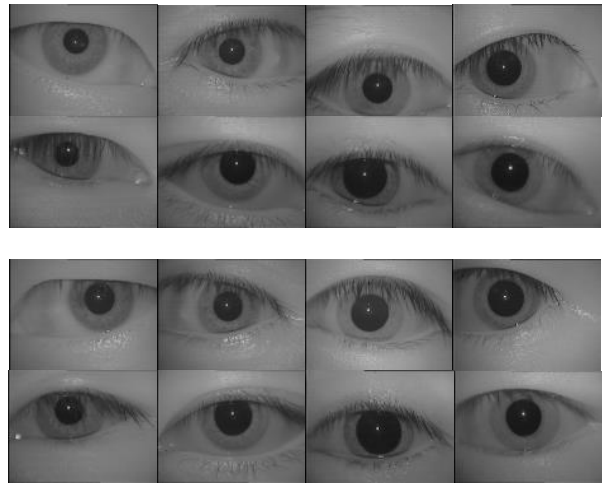

Fig.6. Examples of the left and the right irises.

\section{B. Results and Discussion}

For the evaluation of our biometric system, we must use a database of $\mathrm{N}$ individual having the image of the face and the both instances of the iris. For this, we must use a database which contains the face and the both irises (the left and the right irises). In this work, we chose the SDUMLA-HMT database which includes the biometric traits with the same person captured from the same subject.

Totally, we have seven images of face for each subject with five images for each of his eyes. For each subject and for each modality, the three images are randomly sampled as train samples and the rest are used for the test.

There are several indicators which can use to evaluate the biometric authentication systems. In our work, we choose to use the following indicators: [5]

- FAR is the False Acceptance Rate where the impostors can be accepted wrongly;

- FRR is the False Rejection Rate where the clients 
can be rejected by error;

- EER is the Equal Error Rate. It is the point where FAR is equal to FRR.

- ROC is the Receiver Operating Characteristic Curve. It is the curve which plotted the FRR (in the X-axis) against the FAR (in the Y-axis).

- Accuracy describes the performance of the biometric system.

Figure 7 presents the ROC curve for each database which is a subset extracted from the SDUMLA-HMT database.

We divide the SDUMLA-HMT database into 12 subdatabases. Therefore, each session in face database (Accessory Eye-Glass, Accessory Hat, Expression Frown, Expression close eyes, Expression Smile, Expression Surprise, Illumination One, Illumination Three, Illumination Two, Pose Down, Pose Normal, Pose $\mathrm{Up}$ ) is combined with the iris database to form the databases (BD1, BD2, BD3, BD4, BD5, BD6, BD7, $\mathrm{BD} 8, \mathrm{BD} 9, \mathrm{BD} 10, \mathrm{BD} 11, \mathrm{BD} 12)$ respectively.

- BD1: Accessory eye-glass+ iris database

- BD2: Accessory hat+ iris database

- BD3: Expression frown+ iris database

- BD4: Expression close eyes+ iris database

- BD5: Expression smile+ iris database

- BD6: Expression surprise + iris database

- BD7: Illumination one+ iris database

- BD8: Illumination three+ iris database

- BD9: Illumination two+ iris database

- BD10: Pose down+ iris database

- BD11: Pose normal+ iris database

- BD12: Pose up + iris database.

For each database, each curve plots the FAR in the Yaxis versus the FRR in the $\mathrm{X}$-axis. Each curve represents the performance of the three mono-modal systems tested separately with the SVM classifier (the face recognition, the left iris recognition and the right iris recognition). Also, it demonstrates the influence of each fusion method on the performance of the proposed approach. The first method applied for the fusion is the SVM. However, our contribution is the use of the combination methods coupled with the classification method [35] for the fusion. Therefore, we use the five combination rules (Product, Somme, Min, Max and Mean) coupled with the classification method [35]:

- $\mathrm{CP}$ is the combination of the product rule with the classification method Chikhaoui and Mokhtari [35],

- $\mathrm{CS}$ is the sum rule coupled with the classification method [35],

- $\mathrm{CMI}$ is the combination of the min rule with the classification method [35],

- CMA is the max rule combined with the classification method [35],
- $\mathrm{CME}$ is the mean rule coupled with the classification method [35].

Figure 7 confirms that the multi-biometric is more performance than the mono-modal biometric and it illustrates that the iris modality is more performance than the face modality. Also, it demonstrates that the five fusion approaches (CP, CS, CMI, CMA, and CME) give results better than the SVM method used for the fusion for all the databases.

The execution time of each fusion method (CP, CS, CMI, CMA, and CME) is between $70 \mathrm{sec}$ and $150 \mathrm{sec}$ (Processor: Intel (R) Core (TM) i7-4510U CPU @ $2.00 \mathrm{GHz} 2.60 \mathrm{GHz} \& 4 \mathrm{~GB}$ of memory).

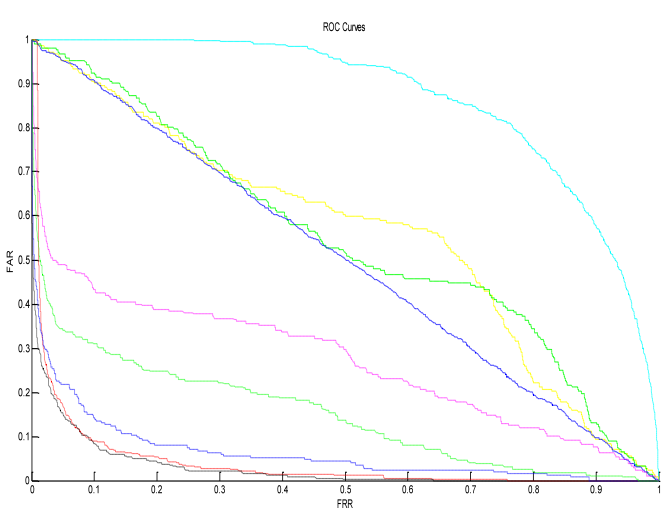

(a)

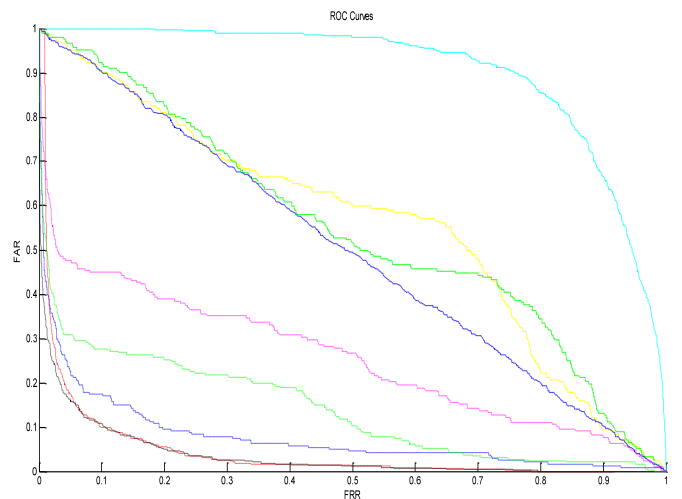

(b)

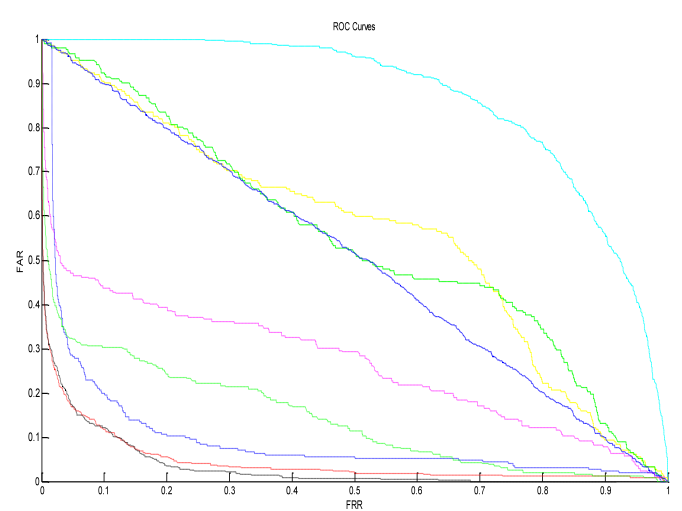

(c) 


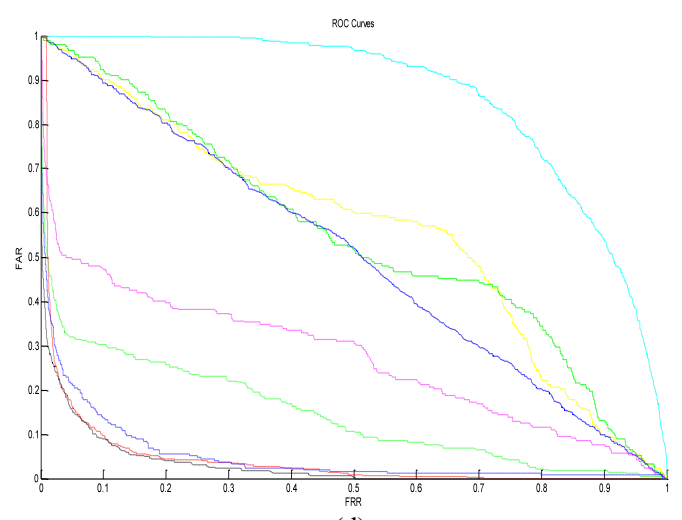

(d)

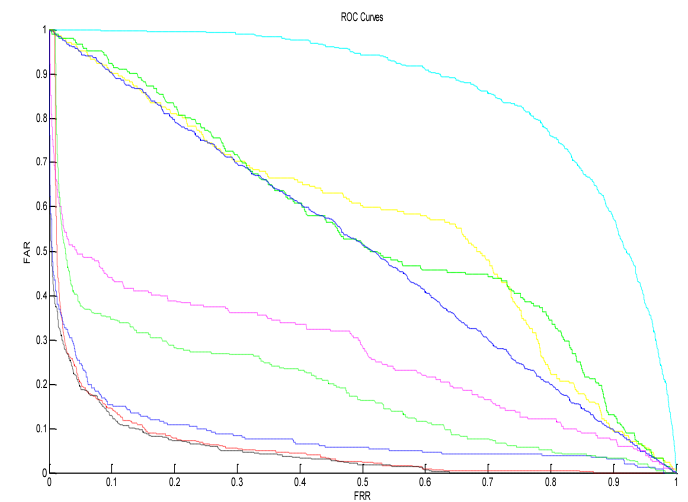

(e)

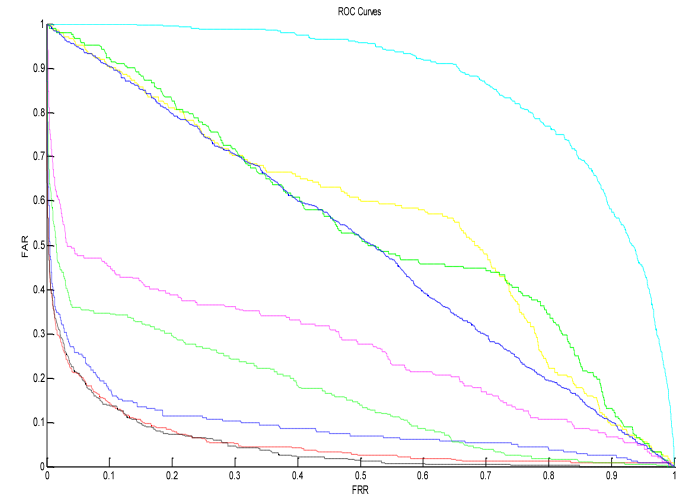

(f)

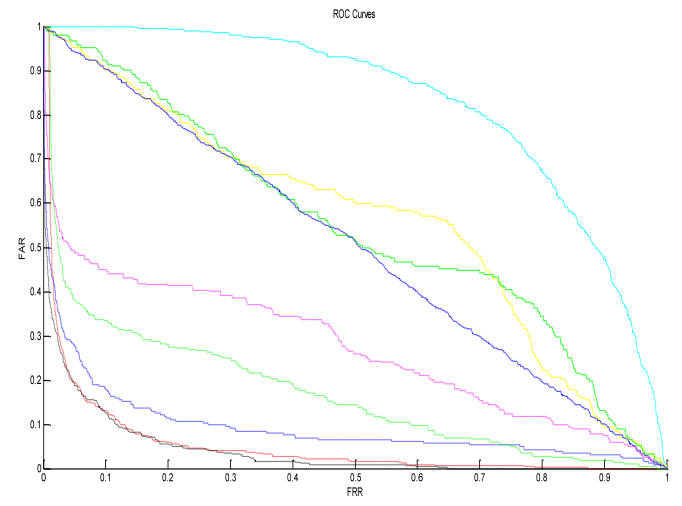

(g)

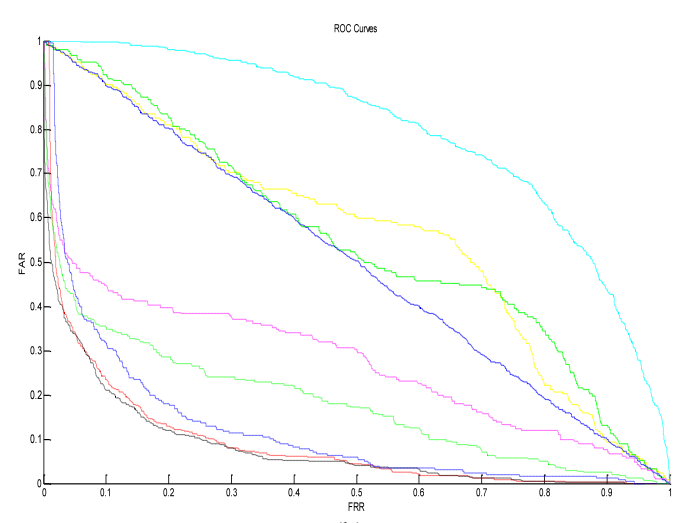

(h)

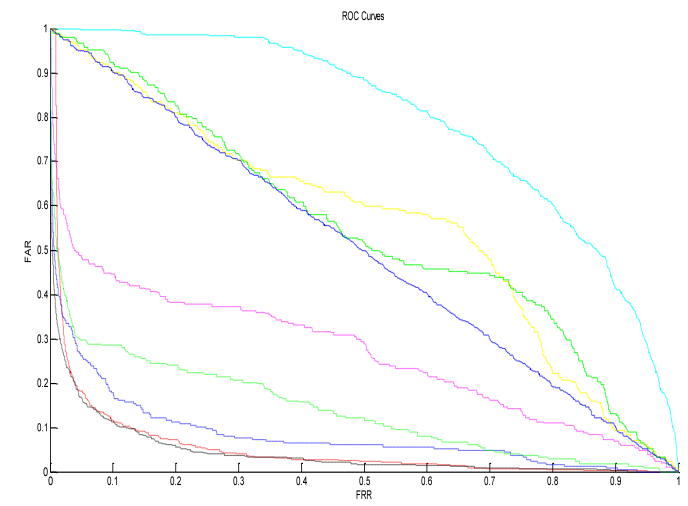

(i)

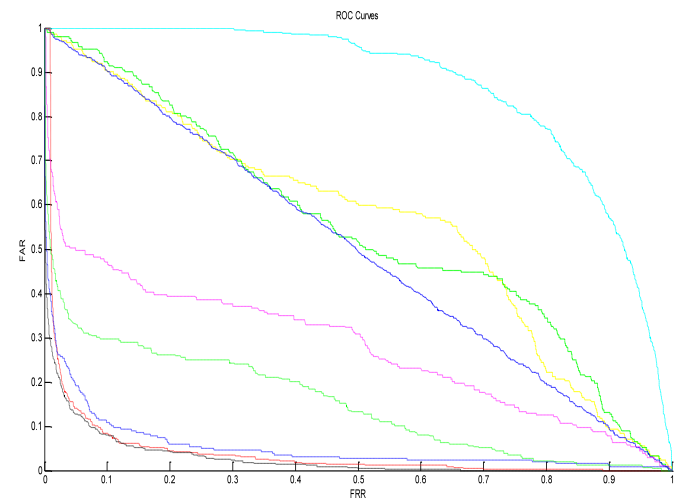

(j)

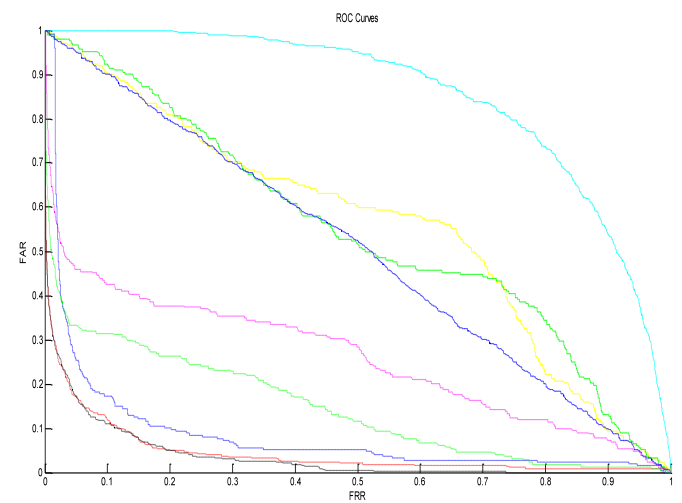

(k) 


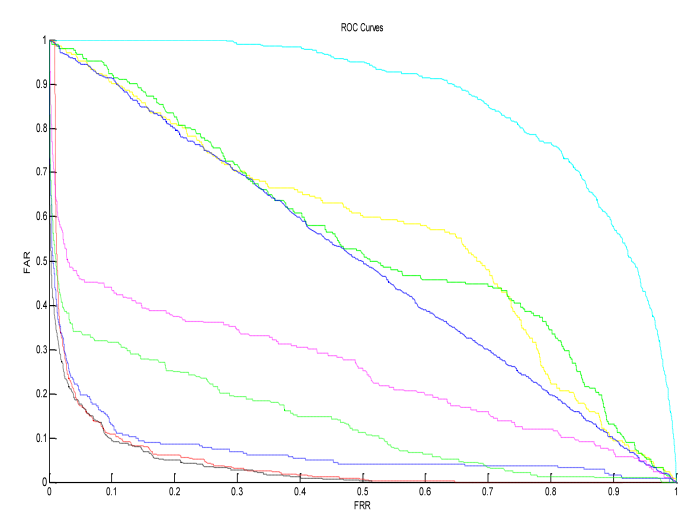

(1)

\begin{tabular}{|l|}
\hline- Face \\
- Left Iris \\
- - Right Iris \\
-- - Fusion:svm \\
$-\cdots-$ - Fusion:Approach(Product) \\
$-\cdots-$ Fusion:Approach(Sum) \\
$-\cdots-$ Fusion:Approach(Min) \\
$-\cdots-$ Fusion:Approach(Max) \\
$-\cdots$ Fusion:Approach(Mean)
\end{tabular}

Fig.7. ROC plot showing the performance of the face recognition, the left iris recognition, the right iris recognition, and the fusion by (SVM, CP, CS, CMI, CMA and CME) on the SDUMLA-HMT

Database:(a):BD1, (b):BD2,(c):BD3, (d):BD4, (e):BD5, (f):BD6, (g):BD7, (h):BD8, (i):BD9, (j):BD10, (k):BD11, (1):BD12 .
For the evaluation of the performance of classification, there are different criteria. Among these criteria, we decided to use the AUC (area under the ROC curve). An area of 1 corresponds to a perfect test; an area of 0.5 signifies a worthless test.

We can obtain from the ROC curve the Equal Error Rate (EER) at which FAR and FRR are equal. Also, we can get the AUC.

Table 1 illustrates the evaluation of the system performance. According to the experiment results, we find that the iris modality is more performance than the face modality. The results show that the fusion gives EER and AUC better than the three mono-modal systems taken separately (the face recognition, the left iris recognition and the right iris recognition). Also, we find that the five approaches of fusion (CP, CS, CMI, CMA, and CME) give results better than the SVM. For all the subdatabases of the SDUMLA-HMT, the both approaches CS and CP give the best result according to the EER and the AUC achieved, then the CMA approach, CME approach, CMI approach respectively with this order.

Table 1. The evaluation of performance using EER and AUC criteria.

\begin{tabular}{|c|c|c|c|c|c|c|c|c|c|c|}
\hline & & \multicolumn{3}{|c|}{ Mono-modal } & \multicolumn{6}{|c|}{ Fusion } \\
\hline & & Face & $\begin{array}{l}\text { Left } \\
\text { iris }\end{array}$ & $\begin{array}{c}\text { Right } \\
\text { iris }\end{array}$ & SVM & $\begin{array}{c}\mathrm{CP} \\
\text { approach }\end{array}$ & $\begin{array}{c}\text { CS } \\
\text { approach }\end{array}$ & $\begin{array}{c}\text { CMI } \\
\text { approach }\end{array}$ & $\begin{array}{c}\text { CMA } \\
\text { approach }\end{array}$ & $\begin{array}{c}\text { CME } \\
\text { approach }\end{array}$ \\
\hline \multirow{2}{*}{ BD1 } & AUC & 0.1338 & 0.4398 & 0.4531 & 0.4999 & 0.9586 & 0.9700 & 0.7291 & 0.9380 & 0.8500 \\
\hline & EER & 0.7827 & 0.5849 & 0.5071 & 0.5016 & 0.0946 & 0.0921 & 0.3558 & 0.1266 & 0.234 \\
\hline \multirow{2}{*}{ BD2 } & AUC & 0.0964 & 0.4398 & 0.4531 & 0.5021 & 0.9557 & 0.9631 & 0.7421 & 0.9237 & 0.8604 \\
\hline & EER & 0.8284 & 0.5849 & 0.5071 & 0.4961 & 0.0991 & 0.1031 & 0.3384 & 0.1459 & 0.2316 \\
\hline \multirow{2}{*}{ BD3 } & AUC & 0.1381 & 0.4398 & 0.4531 & 0.4980 & 0.9573 & 0.9689 & 0.7269 & 0.9499 & 0.8548 \\
\hline & EER & 0.7738 & 0.5849 & 0.5071 & 0.5079 & 0.0991 & 0.0927 & 0.3506 & 0.1199 & 0.2376 \\
\hline \multirow{2}{*}{ BD4 } & AUC & 0.1349 & 0.4398 & 0.4531 & 0.4958 & 0.9547 & 0.9659 & 0.7323 & 0.9041 & 0.8587 \\
\hline & EER & 0.7807 & 0.5849 & 0.5071 & 0.5072 & 0.1108 & 0.1108 & 0.3519 & 0.1394 & 0.2322 \\
\hline \multirow{2}{*}{ BD5 } & AUC & 0.1383 & 0.4398 & 0.4531 & 0.4970 & 0.9408 & 0.9517 & 0.7308 & 0.9173 & 0.8128 \\
\hline & EER & 0.7872 & 0.5849 & 0.5071 & 0.5079 & 0.118 & 0.111 & 0.349 & 0.1346 & 0.2698 \\
\hline \multirow{2}{*}{ BD6 } & AUC & 0.1308 & 0.4398 & 0.4531 & 0.4973 & 0.9443 & 0.9524 & 0.7331 & 0.9079 & 0.8394 \\
\hline & EER & 0.7854 & 0.5849 & 0.5071 & 0.5081 & 0.1226 & 0.1227 & 0.3413 & 0.1434 & 0.2628 \\
\hline \multirow{2}{*}{ BD7 } & AUC & 0.1754 & 0.4398 & 0.4531 & 0.4990 & 0.9483 & 0.9593 & 0.7265 & 0.9078 & 0.8313 \\
\hline & EER & 0.7524 & 0.5849 & 0.5071 & 0.5047 & 0.1156 & 0.1108 & 0.3639 & 0.1439 & 0.2624 \\
\hline \multirow{2}{*}{ BD8 } & AUC & 0.2076 & 0.4398 & 0.4531 & 0.5015 & 0.9110 & 0.9193 & 0.7272 & 0.8807 & 0.8196 \\
\hline & EER & 0.7193 & 0.5849 & 0.5071 & 0.5027 & 0.1604 & 0.1544 & 0.3571 & 0.1875 & 0.2564 \\
\hline \multirow{2}{*}{ BD9 } & AUC & 0.2071 & 0.4398 & 0.4531 & 0.5017 & 0.9466 & 0.9565 & 0.7319 & 0.9175 & 0.8611 \\
\hline & EER & 0.7058 & 0.5849 & 0.5071 & 0.4992 & 0.1108 & 0.1061 & 0.3522 & 0.1452 & 0.2242 \\
\hline \multirow{2}{*}{ BD10 } & AUC & 0.1322 & 0.4398 & 0.4531 & 0.5009 & 0.9590 & 0.9714 & 0.7211 & 0.9468 & 0.8477 \\
\hline & EER & 0.7854 & 0.5849 & 0.5071 & 0.4989 & 0.0918 & 0.0873 & 0.3549 & 0.1055 & 0.25 \\
\hline \multirow{2}{*}{ BD11 } & AUC & 0.1473 & 0.4398 & 0.4531 & 0.4969 & 0.9545 & 0.9635 & 0.7396 & 0.9156 & 0.8537 \\
\hline & EER & 0.7736 & 0.5849 & 0.5071 & 0.5118 & 0.1085 & 0.1061 & 0.3398 & 0.133 & 0.2448 \\
\hline \multirow{2}{*}{ BD12 } & AUC & 0.1342 & 0.4398 & 0.4531 & 0.5016 & 0.9555 & 0.9652 & 0.7460 & 0.9302 & 0.8617 \\
\hline & EER & 0.7791 & 0.5849 & 0.5071 & 0.5 & 0.1061 & 0.0991 & 0.3317 & 0.1107 & 0.2396 \\
\hline
\end{tabular}




\section{CONCLUSION}

This work presents a multi-biometric authentication technique based on fusing two common biometric modalities which are: the face and the both irises (the left and the right irises). Our contribution is the use of a modified method for the iris segmentation and we fused the both modalities by combining two methods the combination method with the classification method. According to the results obtained, we confirmed that the integration of the biometric information gives the best result compared with the use of one biometric modality and we also affirmed the value of combining the face with the both units of the iris modalities.

As future work, we can add other modalities. Also, we can combine different techniques to improve the performance of the authentication system and as possible as we will use artificial neural network approach with deep learning technique to perform results and accuracy of multi-biometric authentication.

\section{REFERENCES}

[1] A. Ross and A. k. Jain, "Multimodal Biometrics: An Overview", in proceedings of 12 the European Signal Processing Conference (EUSIPCO), Vienna, Austria, pp. 1221-1224, Sep. 2004.

[2] V. K. N. Kumar and B. Srinivasan, "New Biometric Approaches for Improved Person Identification Using Facial Detection", International Journal on Image, Graphics and Signal Processing, vol. 4, no. 8, pp. 43-49, Aug. 2012.

[3] V. K. N. Kumar, "Performance of Personal Identification System Technique Using Iris Biometrics Technology", International Journal on Image, Graphics and Signal Processing, vol. 5, no. 5, pp. 63-71, Apr. 2013.

[4] V. R. E. Chirchi and L. M. Waghmare, "Iris Biometric Authentication used for Security Systems", International Journal of Image, Graphics and Signal Processing, vol. 6, no. 9, pp. 54-60, Aug. 2014

[5] Z. Leila and A. Réda, "Multibiometric Fusion: Left and Right Iris Based Authentication Technique", to be appear in IJIGSP Journal, MECS Press.

[6] A. Rattani and M. Tistarelli, "Robust Multimodal and Multiunit Feature Level Fusion of Face and Iris Biometrics", In International Conference on Biometrics, pp. 960-969, Springer Berlin Heidelberg, Jun. 2009.

[7] B. Son and Y. Lee, "Biometric Authentication System Using Reduced Joint Feature Vector of Iris and Face", International Conference on Audio and Video-Based Biometric Person Authentication(AVBPA 2005), pp. 513522, Springer Berlin Heidelberg, 2005.

[8] J. Lin, J. P. Li, H. Lin, and J. Ming, "Robust Person Identification with Face and Iris by Modified PUM Method", International Conference on Apperceiving Computing and Intelligence Analysis (ICACIA 2009), pp. 321-324, Oct 2009.

[9] J. Y. Gan, J. H. Gao, and J. F. Liu, "Research on Face and Iris Feature Recognition Based on 2DDCT and Kernel Fisher Discriminant Analysis", International Conference on Wavelet Analysis and Pattern Recognition (ICWAPR 2008), vol. 1, pp. 401-405, Aug. 2008.

[10] J. Y. Gan and J. F. Liu, "Fusion and Recognition of Face and Iris Feature Based on Wavelet Feature and KFDA", International Conference on Wavelet Analysis and
Pattern Recognition (ICWAPR 2009), pp. 47-50, Jul. 2009.

[11] K. Fakhar, M. El Aroussi, R. Saadane, M. Wahbi, and D. Aboutajdine, "Fusion of Face and Iris Features Extraction Based on Steerable Pyramid Representation for Multimodal Biometrics", International Conference on Multimedia Computing and Systems (ICMCS 2011), pp. 1-4, Jul. 2011.

[12] C. H. Chen and C. Te Chu, "Fusion of Face and Iris Features for Multimodal Biometrics", International Conference on Biometrics, pp. 571-580. Springer Berlin Heidelberg, 2006.

[13] Z. Wang, E. Wang, S. Wang, and Q. Ding, "Multimodal Biometric System Using Face-Iris Fusion Feature", Journal Of Computers, vol. 6, no. 5, pp. 931-938, May. 2011.

[14] H. B. Kekre, V. A. Bharadi, V. I. Singh, V. Kaul, and B Nemade, "Hybrid Multimodal Biometric Recognition using Kekre's Wavelets, 1D Transforms \& Kekre's Vector Quantization Algorithms Based Feature Extraction of Face \& Iris", International Journal of Computer Applications (IJCA), Special Issue for ACM International Conference ICWET, pp. 29-34, 2011.

[15] D. Sharma and A. Kumar, "An Empirical Analysis Over the Four Different Feature-Based Face and Iris Biometric Recognition Techniques", International Journal of Advanced Computer Science and Applications (IJACSA), vol. 3, no. 10, pp. 76-83, 2012.

[16] S. A. H. Nair, P. Aruna, and M. Vadivukarassi, "PCA Based Image Fusion of Face and Iris Biometric Features", International Journal on Advanced Computer Theory and Engineering (IJACTE), vol. 1, no. 2, pp. 106-112, 2013.

[17] Q. Wang, B. Zhu, Y. Liu, L. Xie, and Y. Zheng, "IrisFace Fusion and Security Analysis Based on Fisher Discriminant", International Journal On Smart Sensing and Intelligent Systems, vol. 8, no. 1,pp. 387-407, Mar. 2015.

[18] S. Abuguba, M. M. Milosavljević and N. Maček, "An Efficient Approach to Generating Cryptographic Keys from Face and Iris Biometrics Fused at the Feature Level", International Journal of Computer Science and Network Security (IJCSNS), vol. 15, no. 6, pp. 6-11, Jun. 2015.

[19] Z. Zhang, R. Wang, K. Pan, S. Z. Li, and P. Zhang, "Fusion of Near Infrared Face and Iris Biometrics", International Conference on Biometrics, vol. 4642, pp. 172-180. Springer Berlin Heidelberg, Aug. 2007.

[20] Y. Wang, T. Tan, and A. K. Jain, "Combining Face and Iris Biometrics for Identity Verification", International Conference on Audio- and Video-Based Biometric Person Authentication (AVBPA'03), pp. 805-813, Springer Berlin Heidelberg, Jun. 2003.

[21] N. Morizet and J. Gilles, "A New Adaptive Combination Approach to Score Level Fusion for Face and Iris Biometrics Combining Wavelets and Statistical Moments", Proceedings of the $4^{\text {th }}$ International Symposium on Visual Computing (ISVC '08), pp. 661-671, Springer Berlin Heidelberg, 2008.

[22] X. Zhang, Z. Sun, and T. Tan, "Hierarchical Fusion of Face and Iris for Personal Identification", $20^{\text {th }}$ International Conference on Pattern Recognition (ICPR 2010), pp. 217-220, Aug. 2010.

[23] Y. G. Kim, K. Y. Shin, E. C. Lee, and K. R. Park, "Multimodal Biometric System Based on the Recognition of Face and Both Irises". International Journal of Advanced Robotic Systems, vol. 9, pp. 1-6, 2012.

[24] M. Eskandari and Ö. Toygar, "Person Identification Using Face and Iris Multimodal Biometric System", 
Proceedings of the International Conference on Image Processing, Computer Vision, and Pattern Recognition (IPCV), pp. 1-5, 2012.

[25] M. Eskandari and Ö. Toygar, "Fusion of Face and Iris Biometrics Using Local and Global Feature Extraction Methods", Signal, Image and Video Processing, vol. 8, no. 6, pp. 995-1006, 2014.

[26] M. Eskandari, Ö. Toygar, and H. Demirel, "A New Approach for Face-Iris Multimodal Biometric Recognition Using Score Fusion", International Journal of Pattern Recognition and Artificial Intelligence, vol. 27, no. 3, May. 2013.

[27] H. F. Liau and D. Isa, "Feature Selection for Support Vector Machine Based Face-Iris Multimodal Biometric System", Expert Systems with Applications, vol. 38, no. 9, pp. 11105-11111, Sep. 2011.

[28] M. Vasta, R. Singh, and A. Noore, "Integrating Image Quality in 2v-SVM Biometric Match Score Fusion", International Journal of Neural Systems, vol. 17, no. 5, pp 343-351, 2007.

[29] F. Wang and J. Han, "Multimodal Biometric Authentication Based on Score Level Fusion Using Support Vector Machine", Opto-Electronics Review, vol. 17, no. 1, pp. 59-64, Mar. 2009.

[30] P. A. Johnson, F. Hua, and S. Schuckers, "Comparison of Quality-Based Fusion of Face and Iris Biometrics", International Joint Conference on Biometrics (IJCB), pp. 1-5, Oct. 2011.

[31] N. Wang, L. Lu, G. Gao, F. Wang, and S. Li, "Multibiometrics Fusion Using Aczél-Alsina Triangular Norm", KSII Transactions on Internet and Information Systems(TIIS), vol. 8, no. 7, pp. 2420-2433, Jul. 2014

[32] R. Connaughton, K. W. Bowyer, and P. J. Flynn, "Fusion of Face and Iris Biometrics From a Stand-off Video Sensor", Proceedings of the $22^{\text {nd }}$ Midwest Artificial Intelligence and Cognitive Science Conference (MAICS 2011), pp. 99-106, Cincinatti, OH, Apr. 2011.

[33] R. Connaughton, K. W. Bowyer, and P. J. Flynn, "Fusion of Face and Iris Biometrics", Handbook of Iris Recognition, pp. 219-237, Springer London, 2013.

[34] P. H. Lee, L. J. Chu, Y. P. Hung, S. W. Shih, C. S. Chen, and al., "Cascading Multimodal Verification Using Face, Voice and Iris Information", IEEE International Conference on Multimedia and Expo, pp. 847-850, Jul. 2007.

[35] A. Chikhaoui and A. Mokhtari, "Classification with Support Vector Machines: New Quadratic Programming Algorithm“, COSI’2013, Algiers, Algeria, Jun. 2013.

[36] SDUMLA-HMT Database: http://mla.sdu.edu.cn/sdumlahmt.htm

[37] L. Masek, "Recognition of Human Iris Patterns for Biometric Identification", $P h D$ thesis, University of Western Australia, 2003.

[38] J. Daugman, "High Confidence Recognition of Persons by Rapid Video Analysis of Iris Texture", European Convention on Security and Detection, pp. 244-251, May. 1995.

[39] A. Jain, K. Nandakumar and A. Ross, "Score Normalization in Multimodal Biometric Systems", Pattern Recognition, vol. 38, no. 12, pp. 2270-2285, Dec. 2005.

[40] R. M. Bolle, J. H. Connell and S. Pankanti," Guide to Biometrics", Springer-Verlag, New York, 2004.

[41] A. Chikhaoui, B. Djebbar, and R. Mekki, "New Method for Finding an Optimal Solution to Quadratic Programming Problem”, Journal of Applied Sciences, vol.
10, no. 15, pp. 1627-1631, 2010.

[42] Y. Yin, L. Liu, and X. Sun, "SDUMLA-HMT: A Multimodal Biometric Database", In Chinese Conference on Biometric Recognition (CCBR 2011), Springer Berlin Heidelberg, pp. 260-268, Dec. 2011.

\section{Authors' Profiles}

Leila Zoubida is a Ph.D student in computer science Department at the University of Sidi Bel-Abbes, Algeria. She received a M.S. degree in computer science in 2011 from the Computer Science Department of Tiaret University. Her research interests include the pattern recognition, the biometrics, the image processing and the artificial intelligence.

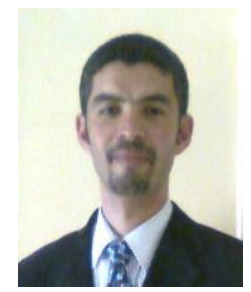

Réda Adjoudj is an associate professor at Sidi Bel-Abbes University. Lastly (in 2014), he obtained his HDR \& in 2006 he received Ph.D in computer science from Djillali Liabes University, Sidi Bel Abbes, and also he earned Master (Magister) from the same university in 2002, and his Engineer degree from the same university in 2000. His major area of research is biometrics, pattern recognition, image processing, and paradigms of artificial intelligence. $\mathrm{He}$ is a member of the EEDIS Laboratory, Computer Science Department.

How to cite this paper: Leila Zoubida, Réda Adjoudj,"Integrating Face and the Both Irises for Personal Authentication", International Journal of Intelligent Systems and Applications (IJISA), Vol.9, No.3, pp.8-17, 2017. DOI: 10.5815/ijisa.2017.03.02 